\title{
Teaching the Geometry of Schemes
}

\author{
Gregory G. Smith and Bernd Sturmfels
}

This chapter presents a collection of graduate level problems in algebraic geometry illustrating the power of Macaulay 2 as an educational tool.

When teaching an advanced subject, like the language of schemes, we think it is important to provide plenty of concrete instances of the theory. Computer algebra systems, such as Macaulay 2, provide students with an invaluable tool for studying complicated examples. Furthermore, we believe that the explicit nature of a computational approach leads to a better understanding of the objects being examined. This chapter presents some problems which we feel illustrate this point of view.

Our examples are selected from the homework of an algebraic geometry class given at the University of California at Berkeley in the fall of 1999. This graduate course was taught by the second author with assistance from the first author. Our choice of problems, as the title suggests, follows the material in David Eisenbud and Joe Harris' textbook The Geometry of Schemes [5].

\section{Distinguished Open Sets}

We begin with a simple example involving the Zariski topology of an affine scheme. This example also indicates some of the subtleties involved in working with arithmetic schemes.

Problem. Let $S=\mathbb{Z}[x, y, z]$ and $X=\operatorname{Spec}(S)$. If $f=x$ and $X_{f}$ is the corresponding basic open subset in $X$, then establish the following:

(1) If $e_{1}=x+y+z, e_{2}=x y+x z+y z$ and $e_{3}=x y z$ are the elementary symmetric functions then the set $\left\{X_{e_{i}}\right\}_{1 \leq i \leq 3}$ is an open cover of $X_{f}$.

(2) If $p_{1}=x+y+z, p_{2}=x^{2}+y^{2}+z^{2}$ and $p_{3}=x^{3}+y^{3}+z^{3}$ are the power sum symmetric functions then $\left\{X_{p_{i}}\right\}_{1 \leq i \leq 3}$ is not an open cover of $X_{f}$.

Solution. (1) To prove that $\left\{X_{e_{i}}\right\}_{1 \leq i \leq 3}$ is an open cover of $X_{f}$, it suffices to show that $e_{1}, e_{2}$ and $e_{3}$ generate the unit ideal in $S_{f}$; see Lemma I-16 in Eisenbud and Harris [5]. This is equivalent to showing that $x^{m}$ belongs to the $S$-ideal $\left\langle e_{1}, e_{2}, e_{3}\right\rangle$ for some $m \in \mathbb{N}$. In other words, the saturation $\left(\left\langle e_{1}, e_{2}, e_{3}\right\rangle: x^{\infty}\right)$ is the unit ideal if and only if $\left\{X_{e_{i}}\right\}_{1 \leq i \leq 3}$ is an open cover of $X_{f}$. We verify this in Macaulay 2 as follows:

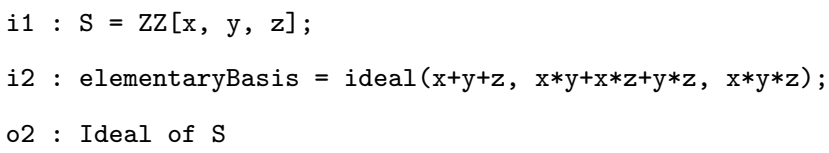




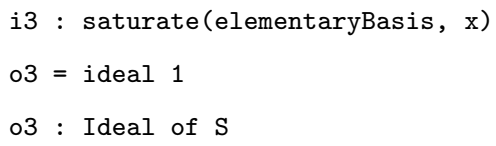

(2) Similarly, to show that $\left\{X_{p_{i}}\right\}_{1 \leq i \leq 3}$ is not an open cover of $X_{f}$, we prove that $\left(\left\langle p_{1}, p_{2}, p_{3}\right\rangle: x^{\infty}\right)$ is not the unit ideal. Calculating this saturation, we find

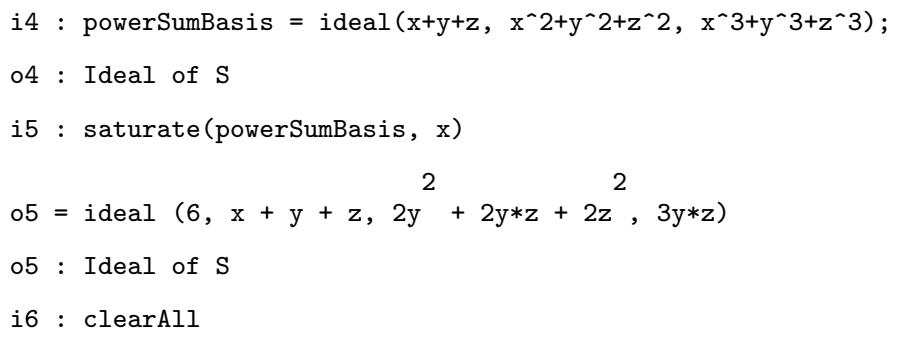

which is not the unit ideal.

The fact that 6 is a generator of the ideal $\left(\left\langle p_{1}, p_{2}, p_{3}\right\rangle: x^{\infty}\right)$ indicates that $\left\{X_{p_{i}}\right\}_{1 \leq i \leq 3}$ does not contain the points in $X$ lying over the points $\langle 2\rangle$ and $\langle 3\rangle$ in $\operatorname{Spec}(\mathbb{Z})$. If we work over a base ring in which 6 is a unit, then $\left\{X_{p_{i}}\right\}_{1 \leq i \leq 3}$ would, in fact, be an open cover of $X_{f}$.

\section{Irreducibility}

The study of complex semisimple Lie algebras gives rise to an important family of algebraic varieties called nilpotent orbits. The next problem examines the irreducibility of a particular nilpotent orbit.

Problem. Let $X$ be the set of nilpotent complex $3 \times 3$ matrices. Show that $X$ is an irreducible algebraic variety.

Solution. A $3 \times 3$ matrix $M$ is nilpotent if and only if its minimal polynomial $p(\mathrm{~T})$ equals $\mathrm{T}^{k}$, for some $k \in \mathbb{N}$. Since each irreducible factor of the characteristic polynomial of $M$ is also a factor of $p(\mathrm{~T})$, it follows that the characteristic polynomial of $M$ is $\mathrm{T}^{3}$. We conclude that the coefficients of the characteristic polynomial of a generic $3 \times 3$ matrix define the algebraic variety $X$.

To prove that $X$ is irreducible over $\mathbb{C}$, we construct a rational parameterization. First, observe that $\mathrm{GL}_{3}(\mathbb{C})$ acts on $X$ by conjugation. Jordan's canonical form theorem implies that there are exactly three orbits; one for each of the following matrices:

$$
N_{(1,1,1)}=\left[\begin{array}{lll}
0 & 0 & 0 \\
0 & 0 & 0 \\
0 & 0 & 0
\end{array}\right], \quad N_{(2,1)}=\left[\begin{array}{lll}
0 & 1 & 0 \\
0 & 0 & 0 \\
0 & 0 & 0
\end{array}\right] \text { and } N_{(3)}=\left[\begin{array}{lll}
0 & 1 & 0 \\
0 & 0 & 1 \\
0 & 0 & 0
\end{array}\right] .
$$

Each orbit is defined by a rational parameterization, so it suffices to show that the closure of the orbit containing $N_{(3)}$ is the entire variety $X$. We demonstrate this as follows: 


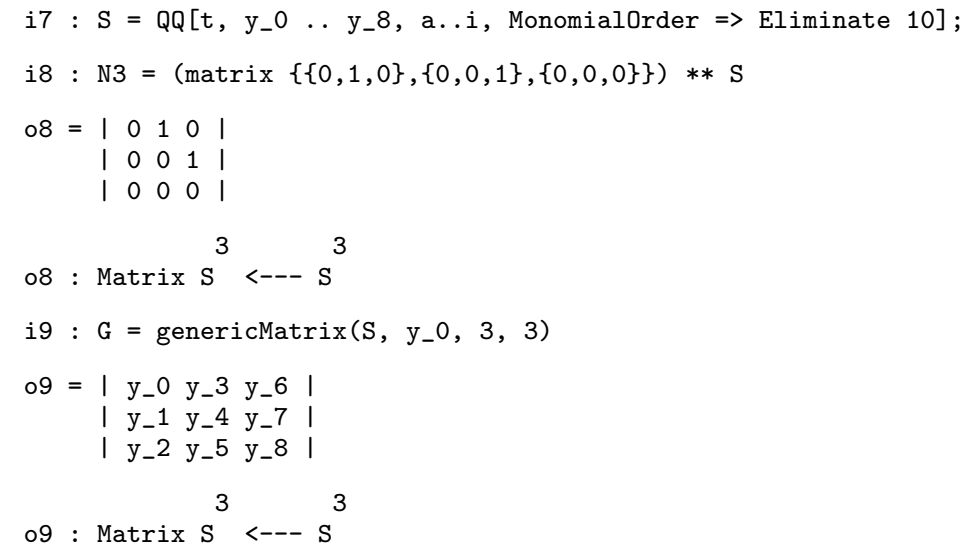

To determine the entries in $G \cdot N_{(3)} \cdot G^{-1}$, we use the classical adjoint to construct the matrix $\operatorname{det}(G) \cdot G^{-1}$.

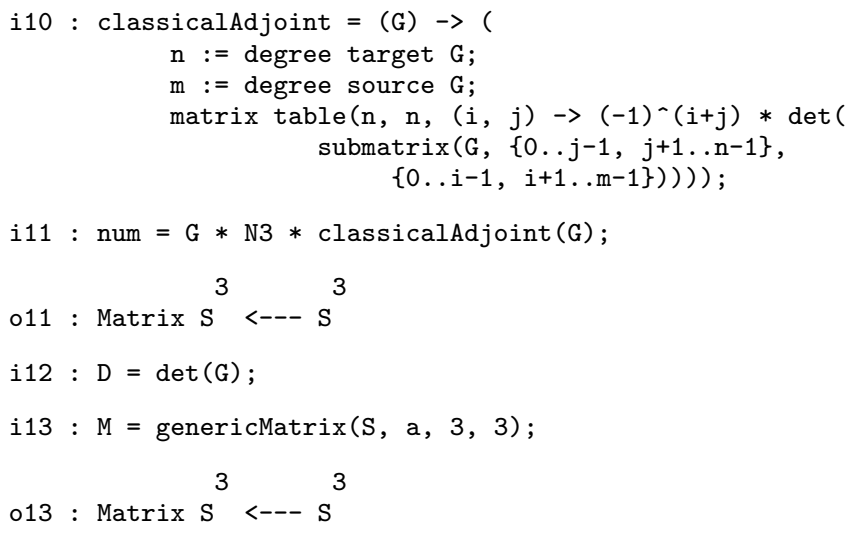

The entries in $G \cdot N_{(3)} \cdot G^{-1}$ give a rational parameterization of the orbit generated by $N_{(3)}$. Using elimination theory - see section 3.3 in Cox, Little and O'Shea [2] - we give an "implicit representation" of this variety.

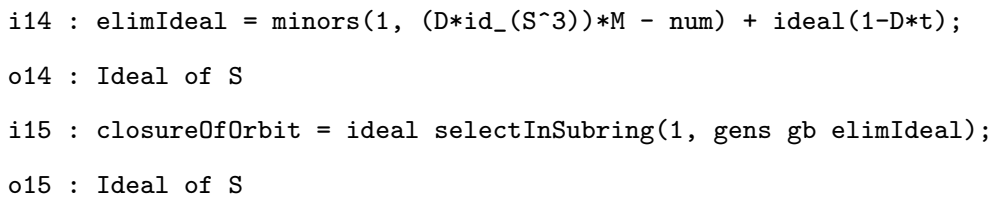

Finally, we verify that this orbit closure equals $X$ scheme-theoretically. Recall that $X$ is defined by the coefficients of the characteristic polynomial of a generic $3 \times 3$ matrix $M$.

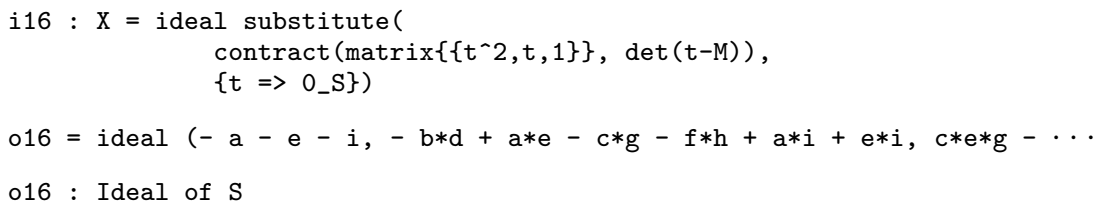




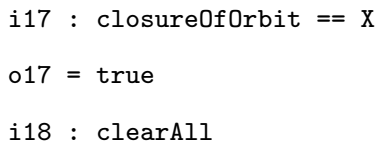

This completes our solution.

More generally, Kostant shows that the set of all nilpotent elements in a complex semisimple Lie algebra form an irreducible variety. We refer the reader to Chriss and Ginzburg [1] for a proof of this result (Corollary 3.2.8) and a discussion of its applications in representation theory.

\section{Singular Points}

In our third question, we study the singular locus of a family of elliptic curves.

Problem. Consider a general form of degree 3 in $\mathbb{Q}[x, y, z]$ :

$$
F=a x^{3}+b x^{2} y+c x^{2} z+d x y^{2}+e x y z+f x z^{2}+g y^{3}+h y^{2} z+i y z^{2}+j z^{3} .
$$

Give necessary and sufficient conditions in terms of $a, \ldots, j$ for the cubic curve Proj $(\mathbb{Q}[x, y, z] /\langle F\rangle)$ to have a singular point.

Solution. The singular locus of $F$ is defined by a polynomial of degree 12 in the 10 variables $a, \ldots, j$. We calculate this polynomial in two different ways.

Our first method is an elementary but time consuming elimination. Carrying it out in Macaulay 2, we have

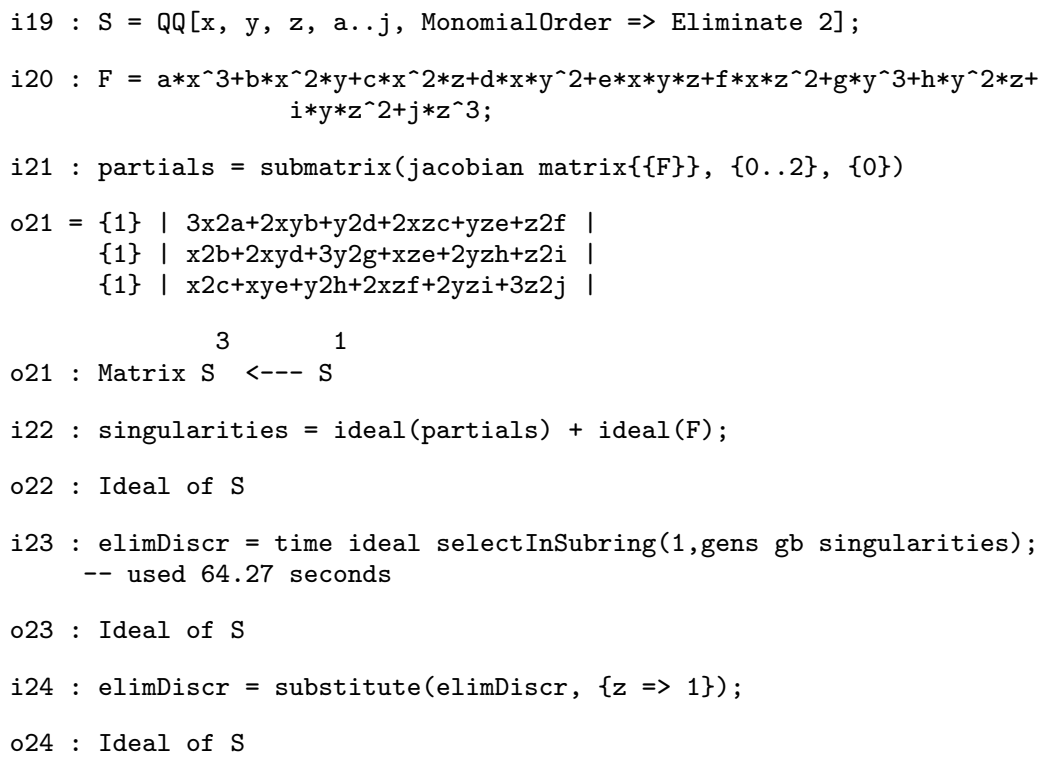


On the other hand, there is also an elegant and more useful determinantal formula for this discriminant; it is a specialization of the formula (2.8) in section 3.2 of Cox, Little and O'Shea [3]. To apply this determinantal formula, we first create the coefficient matrix A of the partial derivatives of $F$.

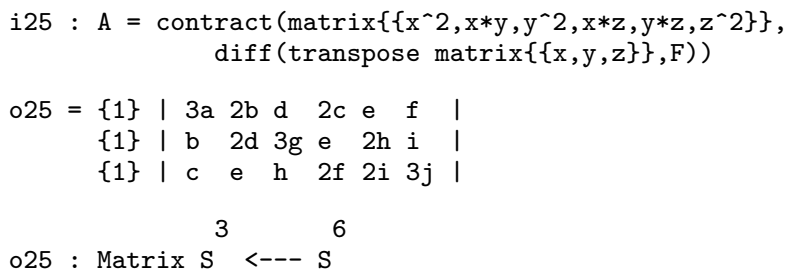

We also construct the coefficient matrix $B$ of the partial derivatives of the Hessian of $F$.

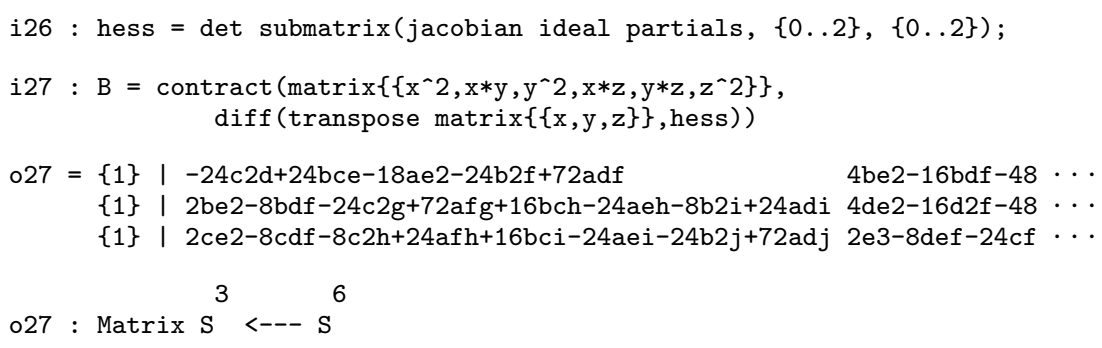

To obtain the discriminant, we combine these two matrices and take the determinant.

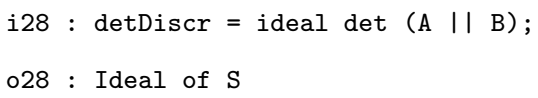

Finally, we check that our two discriminants are equal

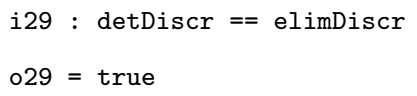

and examine the generator.

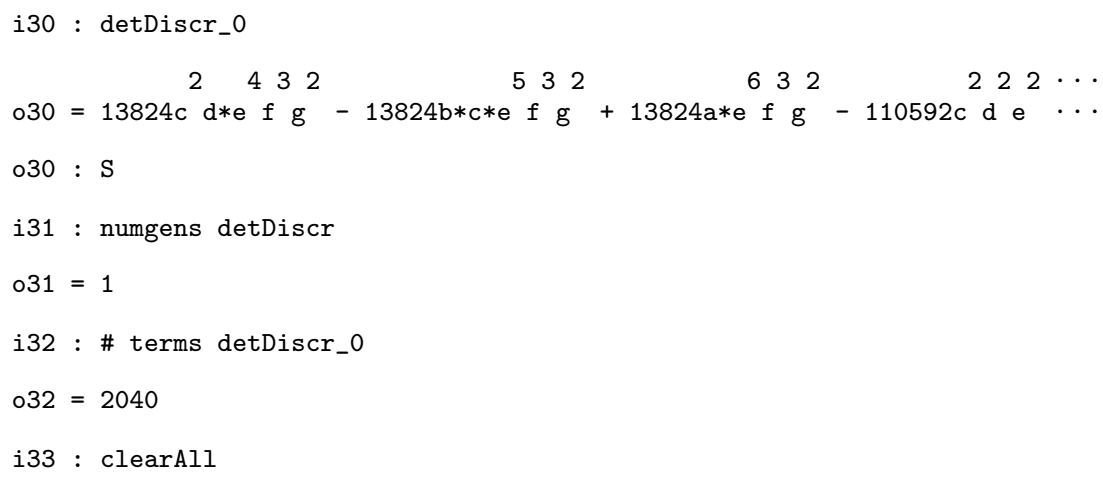

Hence, the singular locus is given by a single polynomial of degree 12 with 2040 terms. 
For a further discussion of singularities and discriminants see Section V.3 in Eisenbud and Harris [5]. For information on resultants and discriminants see Chapter 2 in Cox, Little and O'Shea [3].

\section{Fields of Definition}

Schemes over non-algebraically closed fields arise in number theory. Our fourth problem looks at one technique for working with number fields in Macaulay 2.

Problem (Exercise II-6 in [5]). An inclusion of fields $K \hookrightarrow L$ induces a map $\mathbb{A}_{L}^{n} \rightarrow \mathbb{A}_{K}^{n}$. Find the images in $\mathbb{A}_{\mathbb{Q}}^{2}$ of the following points of $\mathbb{A}_{\mathbb{Q}}^{2}$ under this map.

(1) $\langle x-\sqrt{2}, y-\sqrt{2}\rangle$;

(2) $\langle x-\sqrt{2}, y-\sqrt{3}\rangle$;

(3) $\left\langle x-\zeta, y-\zeta^{-1}\right\rangle$ where $\zeta$ is a 5 -th root of unity;

(4) $\langle\sqrt{2} x-\sqrt{3} y\rangle$;

(5) $\langle\sqrt{2} x-\sqrt{3} y-1\rangle$.

Solution. The images can be determined by using the following three step algorithm: (1) replace the coefficients not contained in $K$ with indeterminates, (2) add the minimal polynomials of these coefficients to the given ideal in $\mathbb{A}_{L}^{2}$, and (3) eliminate the new indeterminates. Here are the five examples:

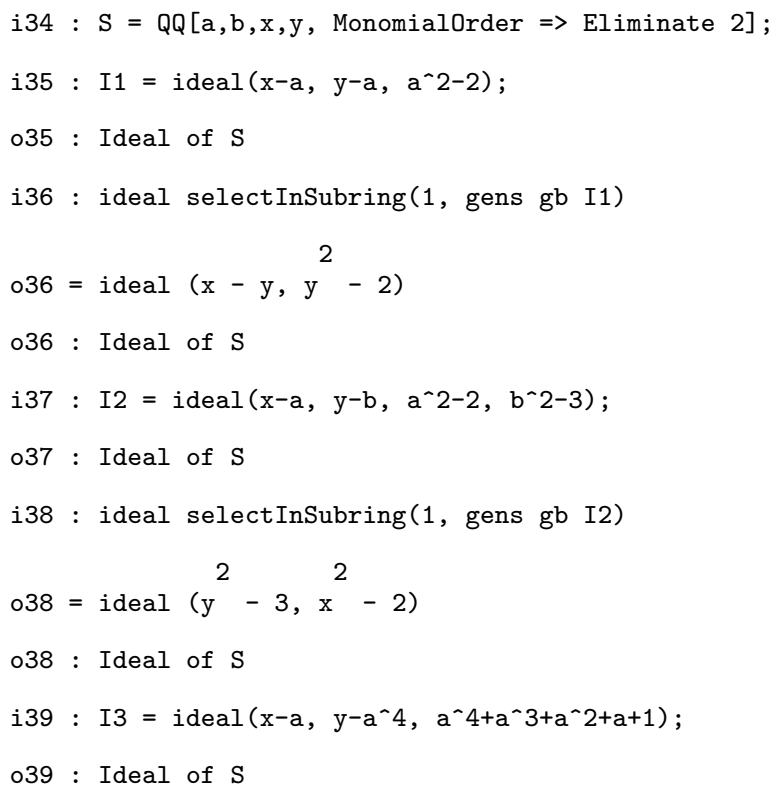




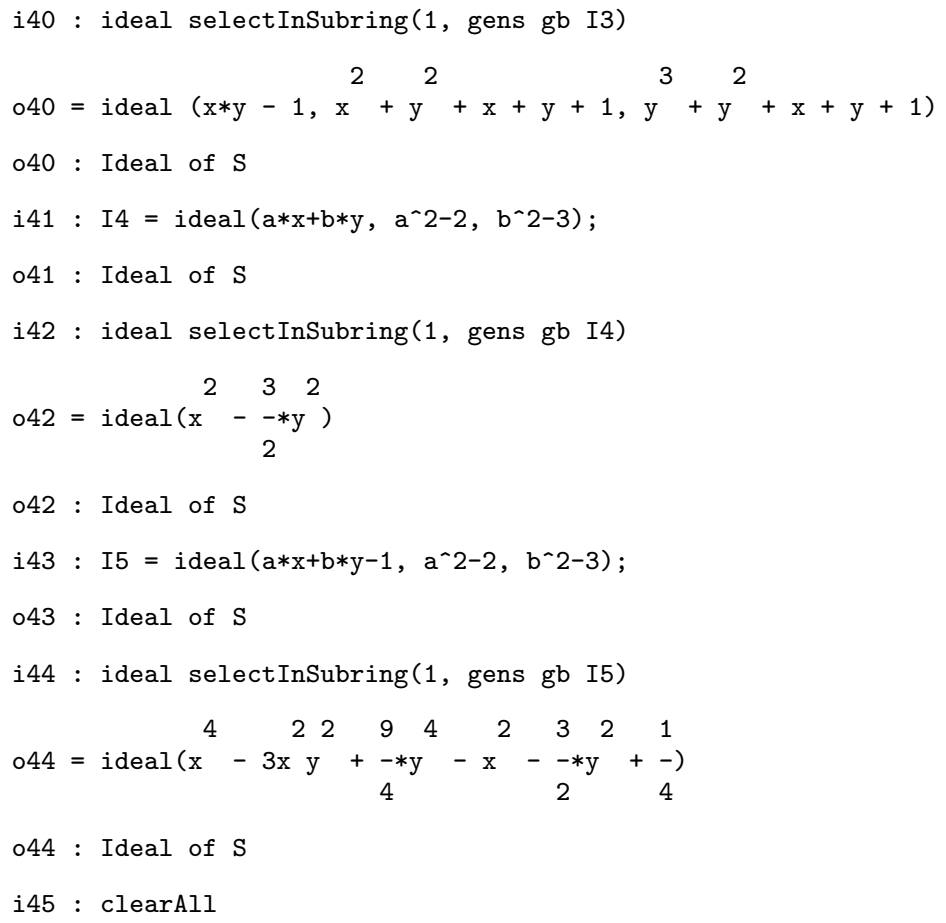

It is worth noting that the points in $\mathbb{A}_{\mathbb{Q}}^{n}$ correspond to orbits of the action of $\operatorname{Gal}(\overline{\mathbb{Q}} / \mathbb{Q})$ on the points of $\mathbb{A} \frac{n}{\mathbb{Q}}$. For more examples and information, see section II.2 in Eisenbud and Harris [5].

\section{$5 \quad$ Multiplicity}

The multiplicity of a zero-dimensional scheme $X$ at a point $p \in X$ is defined to be the length of the local ring $\mathcal{O}_{X, p}$. Unfortunately, we cannot work directly in the local ring in Macaulay 2. What we can do, however, is to compute the multiplicity by computing the degree of the component of $X$ supported at $p$; see page 66 in Eisenbud and Harris [5].

Problem. What is the multiplicity of the origin as a zero of the polynomial equations $x^{5}+y^{3}+z^{3}=x^{3}+y^{5}+z^{3}=x^{3}+y^{3}+z^{5}=0$ ?

Solution. If $I$ is the ideal generated by $x^{5}+y^{3}+z^{3}, x^{3}+y^{5}+z^{3}$ and $x^{3}+y^{3}+z^{5}$ in $\mathbb{Q}[x, y, z]$, then the multiplicity of the origin is

$$
\operatorname{dim}_{\mathbb{Q}} \frac{\mathbb{Q}[x, y, z]_{\langle x, y, z\rangle}}{I \mathbb{Q}[x, y, z]_{\langle x, y, z\rangle}} .
$$


It follows that the multiplicity is the vector space dimension of the ring $\mathbb{Q}[x, y, z] / \varphi^{-1}\left(I \mathbb{Q}[x, y, z]_{\langle x, y, z\rangle}\right)$ where $\varphi: \mathbb{Q}[x, y, z] \rightarrow \mathbb{Q}[x, y, z]_{\langle x, y, z\rangle}$ is the natural map. Moreover, we can express this using ideal quotients:

$$
\varphi^{-1}\left(I \mathbb{Q}[x, y, z]_{\langle x, y, z\rangle}\right)=\left(I:\left(I:\langle x, y, z\rangle^{\infty}\right)\right) .
$$

Carrying out this calculation in Macaulay 2, we obtain:

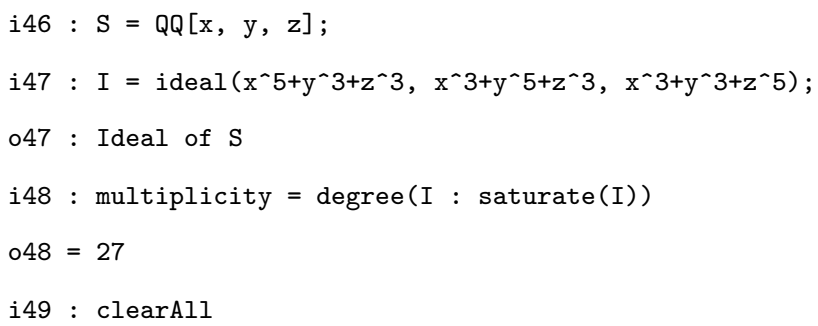

Thus, we conclude that the multiplicity is 27 .

There are algorithms (not yet implemented in Macaulay 2) for working directly in the local ring $\mathbb{Q}[x, y, z]_{\langle x, y, z\rangle}$. We refer the interested reader to Chapter 4 in Cox, Little and O'Shea [3].

\section{$6 \quad$ Flat Families}

Non-reduced schemes arise naturally as flat limits of a family of reduced schemes. Our next problem illustrates how a family of skew lines in $\mathbb{P}^{3}$ gives rise to a double line with an embedded point.

Problem (Exercise III-68 in [5]). Let $L$ and $M$ be the lines in $\mathbb{P}_{k[t]}^{3}$ given by $x=y=0$ and $x-t z=y+t^{2} w=0$ respectively. Show that the flat limit as $t \rightarrow 0$ of the union $L \cup M$ is the double line $x^{2}=y=0$ with an embedded point of degree 1 located at the point $(0: 0: 0: 1)$.

Solution. We first find the flat limit by saturating the intersection ideal and setting $t=0$.

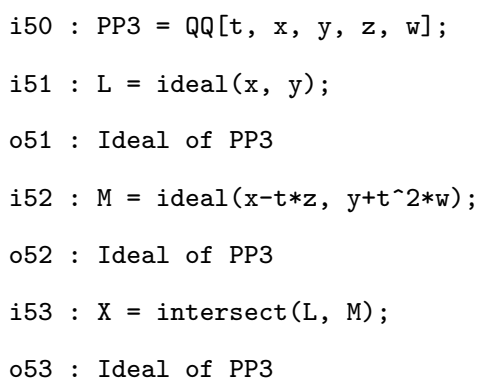




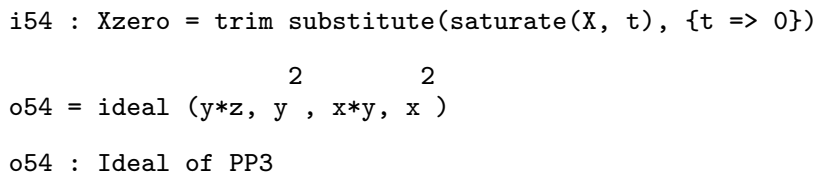

Secondly, we verify that this is the union of a double line and an embedded point of degree 1 .

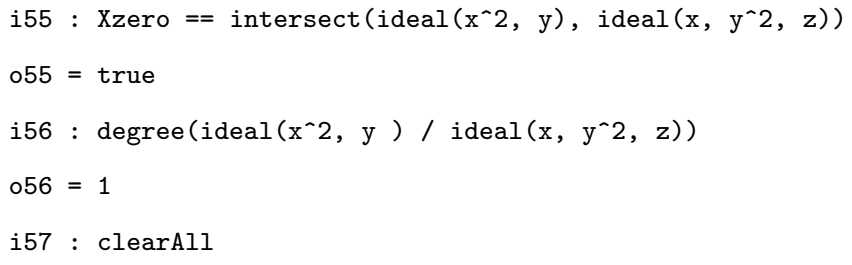

Section III.3.4 in Eisenbud and Harris [5] contains several other interesting limits of various flat families.

\section{Bézout's Theorem}

Bézout's Theorem - Theorem III-78 in Eisenbud and Harris [5] - may fail without the Cohen-Macaulay hypothesis. Our seventh problem is to demonstrate this.

Problem (Exercise III-81 in [5]). Find irreducible closed subvarieties $X$ and $Y$ in $\mathbb{P}^{4}$ such that

$$
\begin{gathered}
\operatorname{codim}(X \cap Y)=\operatorname{codim}(X)+\operatorname{codim}(Y) \\
\operatorname{deg}(X \cap Y)>\operatorname{deg}(X) \cdot \operatorname{deg}(Y) .
\end{gathered}
$$

Solution. We show that the assertion holds when $X$ is the cone over the nonsingular rational quartic curve in $\mathbb{P}^{3}$ and $Y$ is a two-plane passing through the vertex of the cone. First, recall that the rational quartic curve is given by

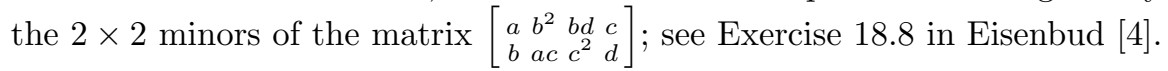
Thus, we have

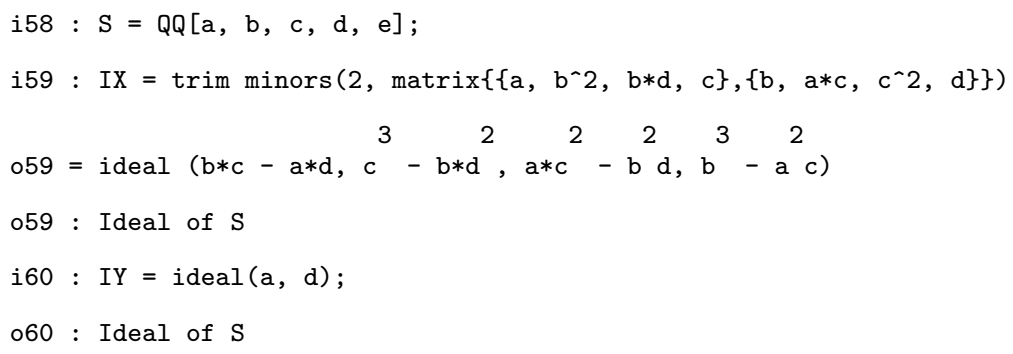




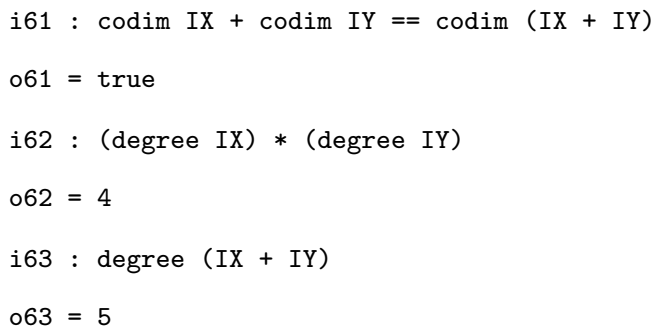

which establishes the assertion.

To understand how this example works, it is enlightening to express $Y$ as the intersection of two hyperplanes; one given by $a=0$ and the other given by $d=0$. Intersecting $X$ with the first hyperplane yields

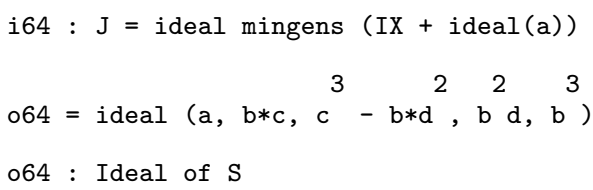

However, this first intersection has an embedded point;

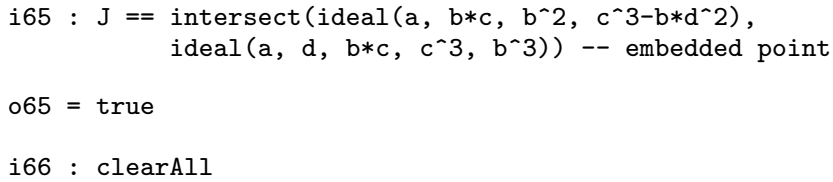

The second hyperplane passes through this embedded point which explains the extra intersection.

\section{Constructing Blow-ups}

The blow-up of a scheme $X$ along a subscheme $Y$ can be constructed from the Rees algebra associated to the ideal sheaf of $Y$ in $X$; see Theorem IV-22 in Eisenbud and Harris [5]. Gröbner basis techniques allow one to express the Rees algebra in terms of generators and relations. We illustrate this method in the next solution.

Problem (Exercises IV-43 \& IV-44 in [5]). Find the blow-up $X$ of the affine plane $\mathbb{A}^{2}=\operatorname{Spec}(\mathbb{Q}[x, y])$ along the subscheme defined by $\left\langle x^{3}, x y, y^{2}\right\rangle$. Show that $X$ is nonsingular and its fiber over the origin is the union of two copies of $\mathbb{P}^{1}$ meeting at a point.

Solution. We first provide a general function which returns the ideal of relations for the Rees algebra. 


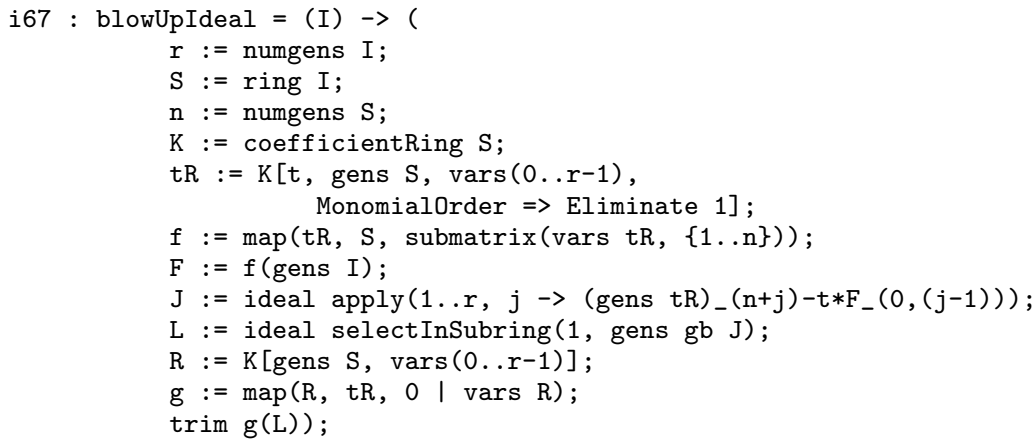

Now, applying the function to our specific case yields:

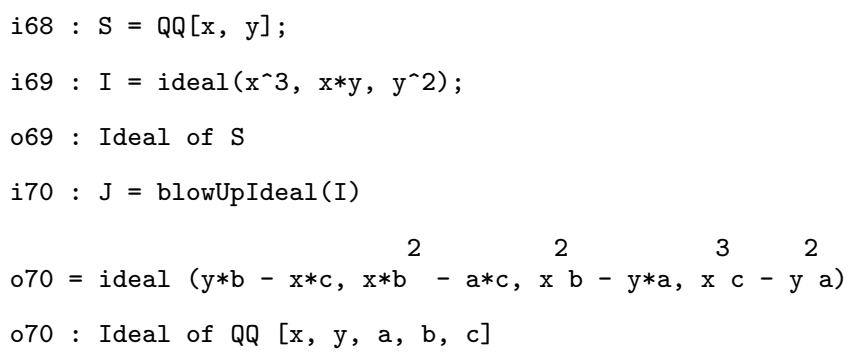

Therefore, the blow-up of the affine plane along the given subscheme is

$$
X=\operatorname{Proj}\left(\frac{(\mathbb{Q}[x, y])[a, b, c]}{\left\langle y b-x c, x b^{2}-a c, x^{2} b-y a, x^{3} c-y^{2} a\right\rangle}\right) .
$$

Using Macaulay 2, we can also verify that the scheme $X$ is nonsingular;

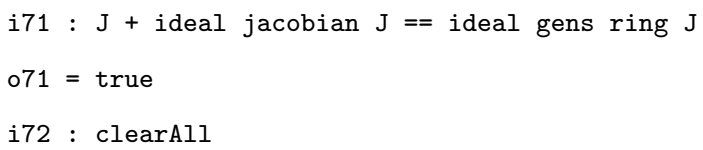

Since we have

$$
\frac{(\mathbb{Q}[x, y])[a, b, c]}{\left\langle y b-x c, x b^{2}-a c, x^{2} b-y a, x^{3} c-y^{2} a\right\rangle} \otimes \frac{\mathbb{Q}[x, y]}{\langle x, y\rangle} \cong \frac{\mathbb{Q}[a, b, c]}{\langle a c\rangle},
$$

the fiber over the origin $\langle x, y\rangle$ in $\mathbb{A}^{2}$ is clearly a union of two copies of $\mathbb{P}^{1}$ meeting at one point. In particular, the exceptional fiber is not a projective space.

Many other interesting blow-ups can be found in section II.2 in Eisenbud and Harris [5].

\section{A Classic Blow-up}

We consider the blow-up of the projective plane $\mathbb{P}^{2}$ at a point. 
Problem. Show that the following varieties are isomorphic.

(a) the image of the rational map from $\mathbb{P}^{2}$ to $\mathbb{P}^{4}$ given by

$$
(r: s: t) \mapsto\left(r^{2}: s^{2}: r s: r t: s t\right) ;
$$

(b) the blow-up of the plane $\mathbb{P}^{2}$ at the point $(0: 0: 1)$;

(c) the determinantal variety defined by the $2 \times 2$ minors of the matrix $\left[\begin{array}{lll}a & c & d \\ b & d & e\end{array}\right]$ where $\mathbb{P}^{4}=\operatorname{Proj}(k[a, b, c, d, e])$.

This surface is called the cubic scroll in $\mathbb{P}^{4}$.

Solution. We find the ideal in part (a) by elimination theory.

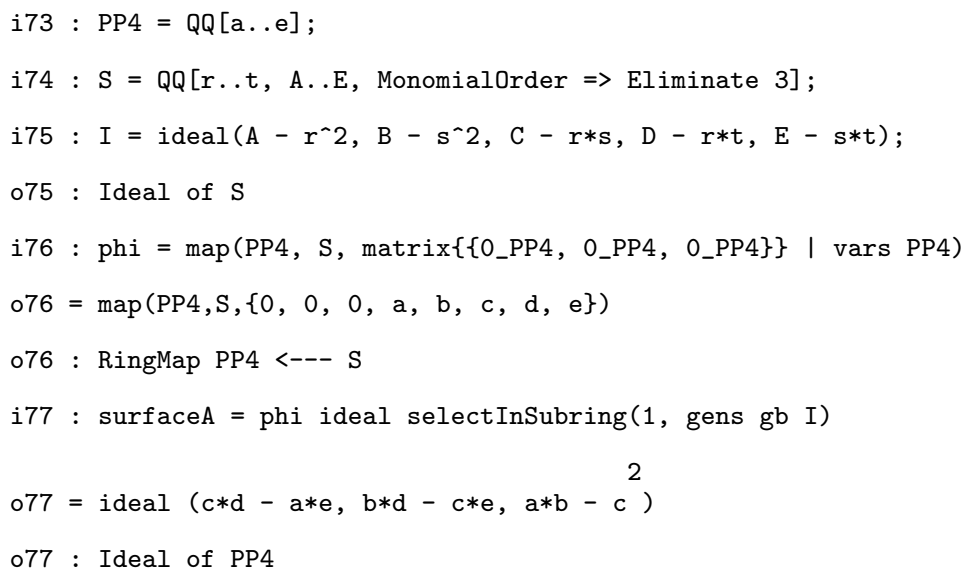

Next, we determine the surface in part $(b)$. We construct the ideal defining the blow-up of $\mathbb{P}^{2}$

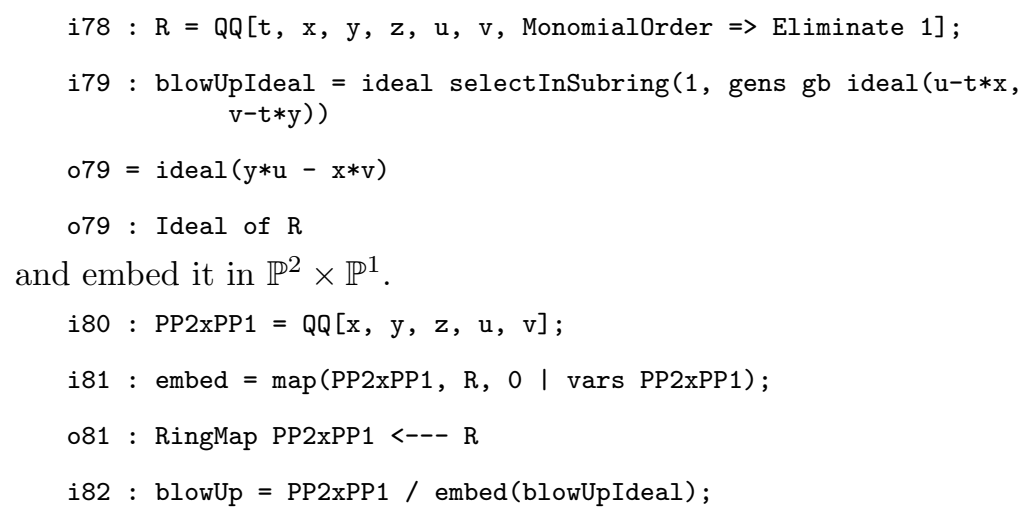

We then map this surface into $\mathbb{P}^{5}$ using the Segre embedding. i83 : PP5 = QQ $[A \ldots F]$; 


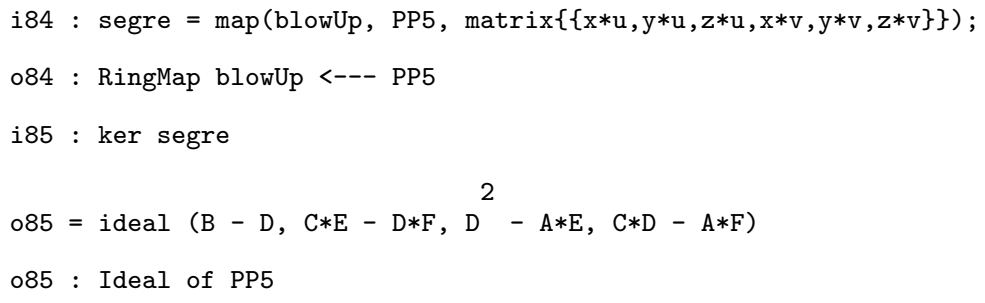

Note that the image under the Segre map lies on a hyperplane in $\mathbb{P}^{5}$. To get the desired surface in $\mathbb{P}^{4}$, we project

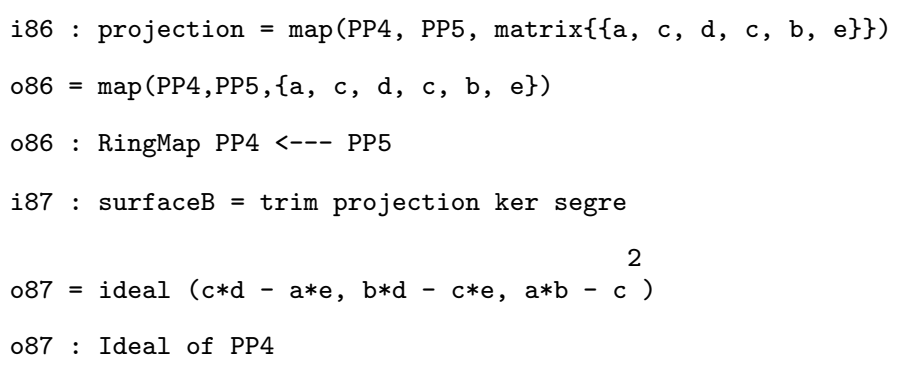

Finally, we compute the surface in part $(c)$.

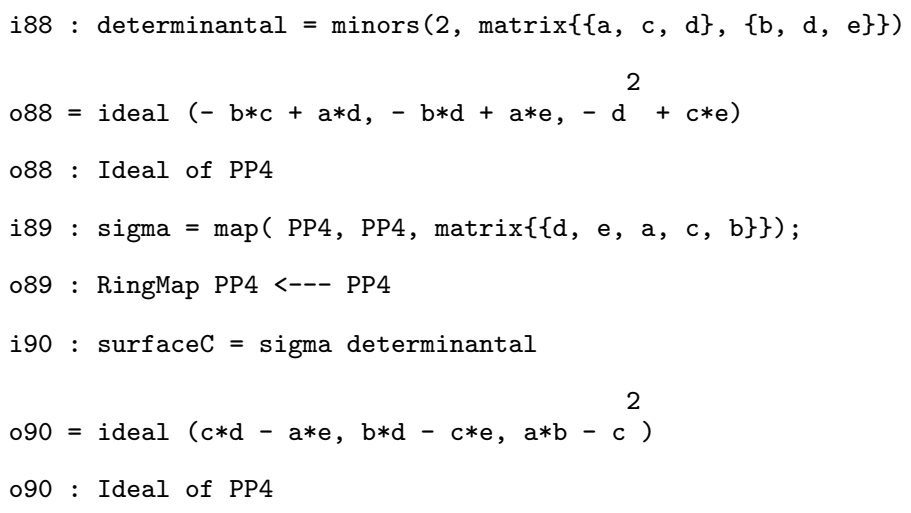

By incorporating a permutation of the variables into definition of surfaceC, we obtain the desired isomorphisms

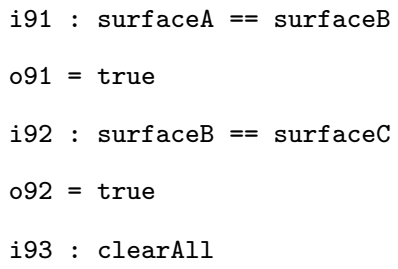

which completes the solution.

For more information of the geometry of rational normal scrolls, see Lecture 8 in Harris [6]. 


\section{Fano Schemes}

Our final example concerns the family of Fano schemes associated to a flat family of quadrics. Recall that the $k$-th Fano scheme $F_{k}(X)$ of a scheme $X \subseteq$ $\mathbb{P}^{n}$ is the subscheme of the Grassmannian parametrizing $k$-planes contained in $X$.

Problem (Exercise IV-69 in [5]). Consider the one-parameter family of quadrics tending to a double plane with equation

$$
Q=V\left(t x^{2}+t y^{2}+t z^{2}+w^{2}\right) \subseteq \mathbb{P}_{\mathbb{Q}[t]}^{3}=\operatorname{Proj}(\mathbb{Q}[t][x, y, z, w])
$$

What is the flat limit of the Fano schemes $F_{1}\left(Q_{t}\right)$ ?

Solution. We first compute the ideal defining $F_{1}\left(Q_{t}\right)$, the scheme parametrizing lines in $Q$.

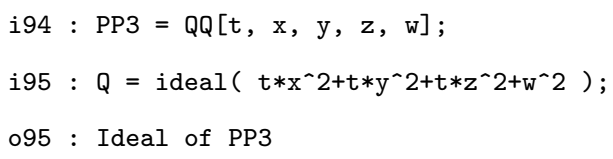

To parametrize a line in our projective space, we introduce indeterminates $u, v$ and $A, \ldots, H$.

i96: $\mathrm{R}=\mathrm{QQ}[\mathrm{t}, \mathrm{u}, \mathrm{v}, \mathrm{A} \ldots \mathrm{H}]$;

We then make a map phi from PP3 to $\mathrm{R}$ sending the variables to the coordinates of the general point on a line.

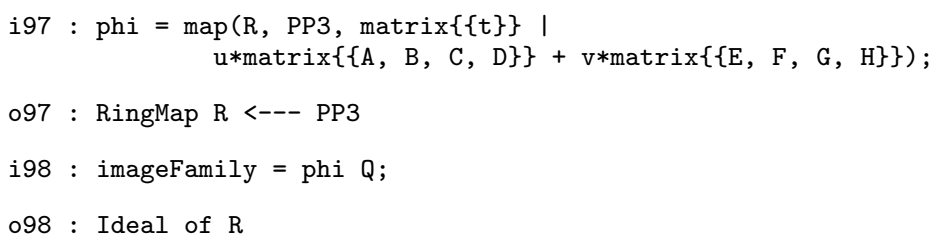

For a line to belong to $Q$, the imageFamily must vanish identically. In other words, $F_{1}(Q)$ is defined by the coefficients of the generators of imageFamily.

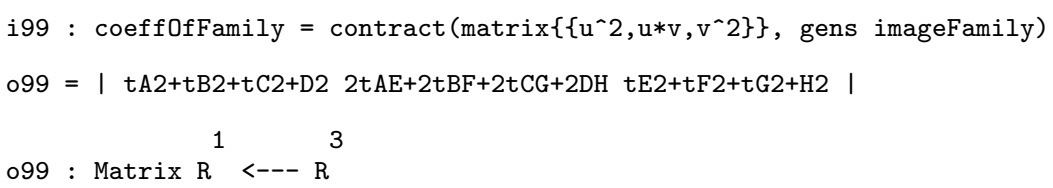

Since we don't need the variables $u$ and $v$, we get rid of them.

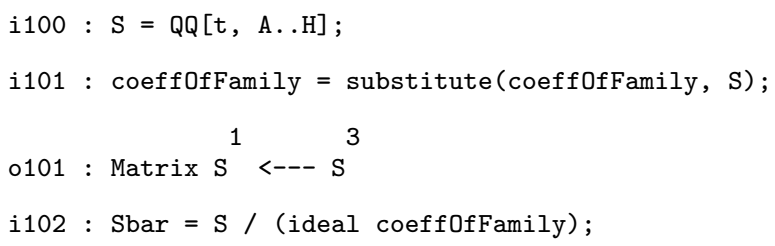


Next, we move to the Grassmannian $\mathbb{G}(1,3) \subset \mathbb{P}^{5}$. Recall the homogeneous coordinates on $\mathbb{P}^{5}$ correspond to the $2 \times 2$ minors of a $2 \times 4$ matrix. We obtain these minors using the exteriorPower function in Macaulay 2.

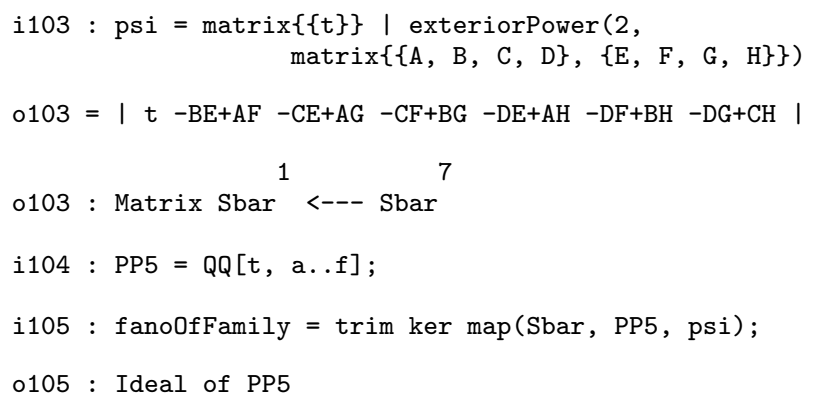

Now, to answer the question, we determine the limit as $t$ tends to 0 .

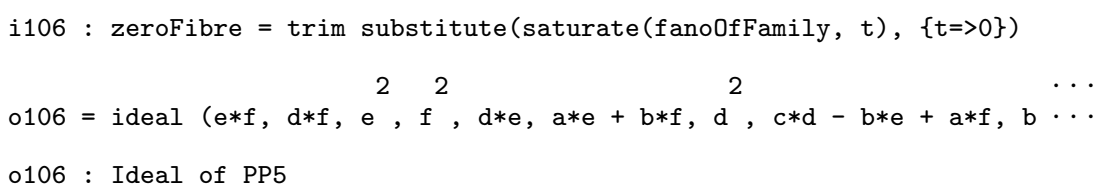

Let's transpose the matrix of generators so all of its elements are visible on the printed page.

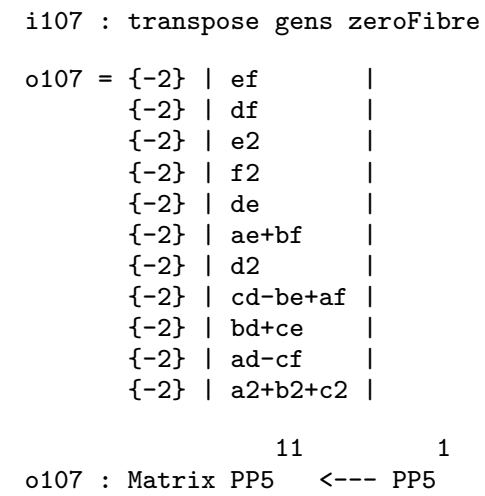

We see that $F_{1}\left(Q_{0}\right)$ is supported on the plane conic $\left\langle d, e, f, a^{2}+b^{2}+c^{2}\right\rangle$. However, $F_{1}\left(Q_{0}\right)$ is not reduced; it has multiplicity two. On the other hand, the generic fiber is

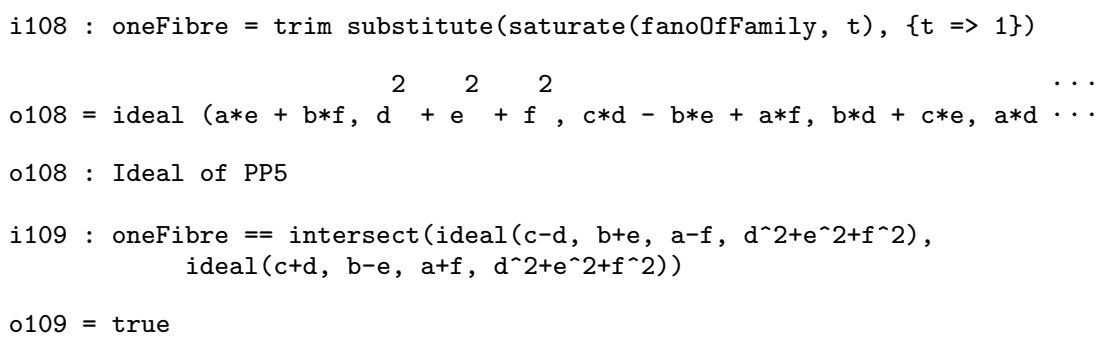


Hence, for $t \neq 0, F_{1}\left(Q_{t}\right)$ is the union of two conics lying in complementary planes and $F_{1}\left(Q_{0}\right)$ is the double conic obtained when the two conics move together.

\section{References}

1. Neil Chriss and Victor Ginzburg: Representation theory and complex geometry. Birkhäuser Boston Inc., Boston, MA, 1997.

2. David Cox, John Little, and Donal O'Shea: Ideals, varieties, and algorithms. Springer-Verlag, New York, second edition, 1997. An introduction to computational algebraic geometry and commutative algebra.

3. David Cox, John Little, and Donal O'Shea: Using algebraic geometry. SpringerVerlag, New York, 1998.

4. David Eisenbud: Commutative algebra with a view toward algebraic geometry. Springer-Verlag, New York, 1995.

5. David Eisenbud and Joe Harris: The geometry of schemes. Springer-Verlag, New York, 2000.

6. Joe Harris: Algebraic geometry, A first course. Springer-Verlag, New York, 1995. 


\section{Index}

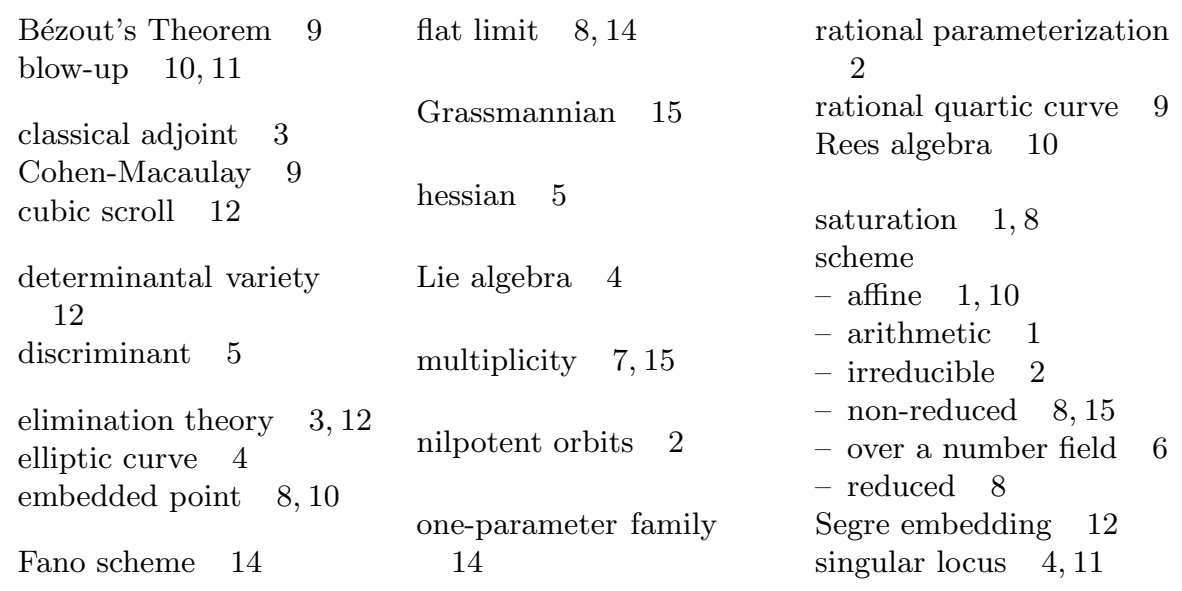

